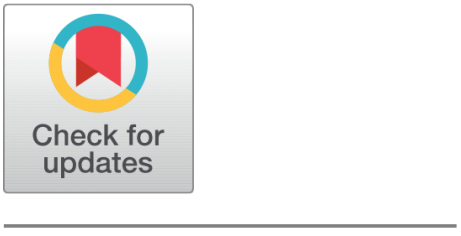

OPEN ACCESS

Received: 19.08.2021

Accepted: 27.11.2021

Published: 27.12.2021

Citation: Srinivas V, Satyanna K (2021) A Result on probabilistic 2-metric space using strong semi compatible mappings. Indian Journal of Science and Technology 14(47): 3467-3474. https://doi.org/ 10.17485/IJST/v14i47.1513

* Corresponding author.

satgjls@gmail.com

Funding: None

Competing Interests: None

Copyright: (c) 2021 Srinivas \& Satyanna. This is an open access article distributed under the terms of the Creative Commons Attribution License, which permits unrestricted use, distribution, and reproduction in any medium, provided the original author and source are credited.

Published By Indian Society for Education and Environment (iSee)

ISSN

Print: 0974-6846

Electronic: 0974-5645

\section{A Result on probabilistic 2-metric space using strong semi compatible mappings}

\author{
V Srinivas ${ }^{1}$, K Satyanna ${ }^{2 *}$ \\ 1 Assistant Professor, Department of Mathematics, University college of Science, Osmania \\ University, 500004, Telangana, India \\ 2 Assistant Professor, Department of Mathematics, M.A.L.D. Government Degree College, \\ Palamoor University, Gadwal, Mahaboobnagar, 509125, Telangana
}

\section{Abstract}

Objective/Aim: To generate a fixed point theorem in probabilistic 2-metric space. Method: By employing strong semi compatible mappings and sub sequentially continuous mappings. Findings: Generated unique common fixed point theorem and substantiated with appropriate example. Novelty/Improvement: The concepts of strong semi compatible mappings and sub sequentially continuous mappings are weaker than existing conditions like weakly compatible mappings which generalizes the theorem of V. K. Gupta, Arihant Jain and Rajesh kumar.

Keywords: Strong semi compatible; sub sequentially continuous; Probabilistic 2 metric space; conditional semi compatible; conditional compatible

\section{Introduction}

The analysis is main branch of mathematics, one of its main ingredient is to give the solution of problems in all fields which is based on fixed points theory. This turned way to extraction of fixed point theorems with minimal effort. Menger ${ }^{(1)}$ made ground stone to generate probabilistic concept for the distance, resulting several theorems. After words many generalizations of commuting mappings came into the fixed point theory ${ }^{(2)}$. The major achievement was the introduction of compatibility concept in Menger space by Mishra ${ }^{(3)}$, resulting the flood of many fixed point theorems established. Some more results were obtained on this area by Martinez et.al ${ }^{(4)}$. In this connection searching of fixed points theorems led to the arrival of the concepts of continuity, reciprocal continuity, sub sequential continuity, semi compatibility, conditional semi compatibility and strong semi compatibility in Menger space like in ${ }^{(5)}$. These concepts have been extended to generate some more results like in ${ }^{(6)}$. Chauhan ${ }^{(7)}$ introduced the notion of occasionally weakly compatible maps in megner space. In this context many researchers are focusing on the existence of fixed point theorems and their applications in different ways ${ }^{(8)}$. Mukesh Kumar Jain and Mohammad Saeed Khan ${ }^{(9)}$ coined the new concept strong semi compatible mappings and obtained some results in metric space. Ravindra K. Bisht and Naseer Shahazad ${ }^{(10)}$ used the concept of faintly compatible and extracted some more fixed point theorems in metric space. V.K. Gupta, Arihant jain and Rajesh kumar ${ }^{(11)}$ presented some results on probabilistic 2 - metric space by applying weakly compatible mappings. Recently some results in probabilistic 2 - metric were obtained by using faintly compatible and reciprocally 
continuous, sub sequentially continuous and semi compatible mappings in Menger space ${ }^{(12-14)}$. Further some more theorems witnessed like ${ }^{(15)}$ on C-class functions on an intuitionistic menger space and also functional spaces by A. M. Zidan et.al. ${ }^{(16) \text {. }}$

\section{Materials and Methods}

\subsection{Definition(11)}

$F: R \rightarrow R^{+}$is a distribution function if

1. Non- decreasing

2. Continuous from left

3. $\operatorname{Inf}\{F(t): t \in R\}=0$

4. $\operatorname{Sup}\{F(t): t \in R\}=1$.

Representing the collection of all distributive functions as $D_{f}$.

\subsubsection{Example}

Special distributive function $H$ is defined by

$$
H(x)=\left\{\begin{array}{l}
1, x>1 \\
0, x \leq 1
\end{array}\right.
$$

\subsection{Definition ${ }^{(11)}$}

An ordered pair $(X, F)$ is known as probabilistic 2-metric space (2-PM space) where $X \neq \phi$ is an arbitrary set and $F$ is mapping withF $: X \times X \times X \rightarrow D_{f}$ here $D_{f}$ is the set of all distribution functions, where the function $\mathrm{F}$ takes the value at $(a, b, c) \in X \times X \times X$ is denoted by $F_{a, b, c}$ satisfying

$$
\begin{aligned}
& F_{a, b, c}(0)=0 \\
& \forall \mathrm{b}, \mathrm{c} \in \mathrm{Xb} \neq c, \exists \mathrm{a} \in \mathrm{X} \text { with } F_{a, b, c}\left(t_{\phi}\right)<1 \text { such that } t_{\phi}>0 \\
& F_{a, b, c}\left(t_{\phi}\right)=1 \forall t_{\phi}>0 \text { when } \mathrm{a}=\mathrm{b} \text { or } \mathrm{b}=\mathrm{c} \text { or } \mathrm{c}=\mathrm{a} \\
& F_{a, b, c}\left(t_{\phi}\right)=F_{b, c, a}\left(t_{\phi}\right)=F_{c . a, b}\left(t_{\phi}\right) \\
& F_{a, b, c}\left(t_{u}\right)=F_{b, c, a}\left(t_{v}\right)=F_{c, a, b}\left(t_{w}\right)=1 \text { then } F_{a, b, c}\left(t_{u}+t_{v}+t_{w}\right)=1 \\
& \forall \mathrm{a}, \mathrm{b}, \mathrm{c} \in \mathrm{X} \text { and } t_{u}, t_{v}, t_{w} \geq 0 .
\end{aligned}
$$

\subsection{Definition $^{(11)}$}

The mapping t: $[0,1]^{3} \rightarrow[0,1]$ is called t-norm if

$$
\begin{aligned}
& \mathrm{t}(\mathrm{a}, 1,1)=\mathrm{a}, \mathrm{t}(0,0,0)=0 \\
& \mathrm{t}(a, b, c)=\mathrm{t}(b, c, a)=\mathrm{t}(c, a, b) \\
& \mathrm{t}\left(a_{1}, b_{1}, c_{1}\right) \geq \mathrm{t}\left(a_{2}, b_{2}, c_{2}\right) \text { for } a_{1} \geq a_{2}, b_{1} \geq b_{2}, c_{1} \geq c_{2} \\
& \mathrm{t}(\mathrm{t}(a, b, \mathrm{w}), \mathrm{p}, \mathrm{q})=\mathrm{t}(a, \mathrm{t}(b, \mathrm{w}, \mathrm{p}), \mathrm{q})=\mathrm{t}(a, b, \mathrm{t}(\mathrm{w}, \mathrm{p}, \mathrm{q})) \\
& \forall \mathrm{a}, \mathrm{b}, \mathrm{c}, p, q, w, a_{i}, b_{i}, b_{i} \in X \text { for } \mathrm{i}=1,2 .
\end{aligned}
$$

\subsubsection{Example}

Let $t(x, y, z)=\min \{x, y, z\}$ defines a t-norm.

\subsection{Definition ${ }^{(11)}$}

A 2-Menger space is $(X, F, t)$ formed by $(X, F)$ and $t$ where $(X, F)$ is a 2-PM space and ' $t$ ' is a t-norm having:

$$
F_{a, b, c}\left(t_{u}+t_{v}+t_{w}\right) \geq \mathrm{t}\left(F_{a, b, d}\left(t_{u}\right)_{m} F_{a, d, c}\left(t_{v}\right), F_{d, b, c}\left(t_{w}\right)\right) \forall \mathrm{a}, \mathrm{b}, \mathrm{c}, \mathrm{d} \in \mathrm{X} a n d t_{u}, t_{v}, t_{w} \geq 0 \text {. }
$$




\subsubsection{Example}

Consider $(X, d)$ be any metric space and $t_{1}$ is a $\mathrm{t}$-norm for each $t_{1} \in[0,1]$, define

$F_{\alpha, \beta, a}\left(t_{1}\right)=\left\{\begin{array}{c}\frac{t_{1}}{t_{1}+d(\alpha, \beta)}, t_{1}>0 \\ 0, t_{1}=1\end{array}\right.$

$\forall \alpha, \beta$, in $\mathrm{X}$ and fixed $a, t_{1}>0$.

Then $(X, F, t)$ forms a 2-PM space.

\subsection{Definition ${ }^{(12)}$}

A sequence in $\left\langle a_{n}\right\rangle$ in $(X, F, t)$ converges to $\beta$ if $\lim _{n \rightarrow \infty} F_{\alpha_{n}, \beta, \alpha}(t)=1$ for all $\mathrm{t}>0$.

\subsection{Definition ${ }^{(12)}$}

A sequence in $\left\langle a_{n}\right\rangle$ in $(X, F, t)$ is cauchy if $\lim _{n \rightarrow \infty} F_{a_{n}}, a_{m}, a(t)=1$ for all $\mathrm{t}>0$.

\subsection{Definition ${ }^{(9)}$}

A 2-Menger space is $(X, F, t)$ is complete if every cauchy sequence converges in $\mathrm{X}$.

\subsection{Definition ${ }^{(4,11)}$}

Self-mappings $\mathrm{P}, \mathrm{S}$ in $(X, F, t)$ is termed as compatible if $\lim _{n \rightarrow \infty} F_{P S x_{n}, S P x_{n}, a}(\beta)=1 \forall \mathrm{a} \in \mathrm{X}$ and $\beta>0$.

\subsection{Definition (11)}

Self-mappings $P, S$ in $(X, F, t)$ are weakly compatible if they are commuting at every coincidence point.

\subsection{Definition $^{(9)}$}

“ The self -mappings $\mathrm{P}$ and $\mathrm{S}$ are conditionally semi compatible if whenever the sequences $\left\langle x_{n}\right\rangle$ satisfying $\left\{\left\langle x_{n}\right\rangle: \lim _{n \rightarrow \infty} P x_{n}=\lim _{n \rightarrow \infty} S x_{n}\right\} \neq \emptyset$, then there exists another sequence $\left\langle t_{n}\right\rangle \in \mathrm{X}$ with $\lim _{n \rightarrow \infty} P t_{n}=\lim _{n \rightarrow \infty} S t_{n}=u$ for some $u \in X$ such that $\lim _{n \rightarrow \infty} F_{P S t_{n}}, s u, a(\beta)=1$ and $\lim _{n \rightarrow \infty} F_{S P t_{n}}, p u, a(\beta)=1 \quad \forall \mathrm{a} \in \mathrm{X} \forall \mathrm{a} \in \mathrm{X}$ and $\beta>0$."

\subsection{Definition $^{(9)}$}

“The self -mappings $\mathrm{P}$ and $\mathrm{S}$ are strongly semi compatible if $\mathrm{P}$ and $\mathrm{S}$ are conditionally semi compatible and $\mathrm{P}$ and $\mathrm{S}$ commute on a nonempty subset of the set of coincidence points, whenever the set of coincidence points is non-empty."

\subsubsection{Example}

Consider $\mathrm{X}=\mathrm{R}, \mathrm{d}$ is usual metric in $\mathrm{X}$ and define $F_{\alpha, \beta, a}\left(t_{1}\right)=\left\{\begin{array}{c}\frac{t_{1}}{t_{1}+(d(\alpha, \beta)}, t_{1}>0 \\ 0, t_{1}=1\end{array}\right.$

$\forall \alpha, \beta$ in $\mathrm{X}$ and fixed $\mathrm{a}, \mathrm{t}_{1}>0$.

Define mappings C, D : X $\rightarrow>\mathrm{X}$ as $C(x)=e^{x} \quad \forall x \in R$ and $D(x)=e^{x^{2}} \forall x \in R$

Choose a sequence $\left\langle t_{m}\right\rangle=1-\frac{11}{m} \quad \forall m \geq 1$. Then

$C\left(t_{m}\right)=C\left(1-\frac{11}{m}\right)=e^{1-\frac{w}{m}} \rightarrow e$ and

$D\left(t_{m}\right)=D\left(1-\frac{11}{m}\right)=e^{\left(1-\frac{11}{m}\right)^{2}} \rightarrow e$ as $m \rightarrow \infty$.

Implies $\left\{\left\langle t_{m}\right\rangle: \lim _{m \rightarrow \infty} C t_{m}=\lim _{m \rightarrow \infty} D t_{m}\right\} \neq \varnothing$.

Then there exists another sequence $\left\langle t_{m}\right\rangle=\frac{3}{5 m} \quad \forall m \geq 1$ Then

$C\left(t_{m}\right)=C\left(\frac{3}{5 m}\right)=e^{\frac{3}{5 m}} \rightarrow 1$

$D\left(t_{m}\right)=D\left(\frac{3}{5 m}\right)=e^{\left(\frac{3}{5 m}\right)^{2}} \rightarrow 1$ as $m \rightarrow \infty$

Further 


$$
\begin{aligned}
& C D\left(t_{m}\right)=C\left(e^{\left(\frac{3}{5 m}\right)^{2}}\right)=e^{e^{\left(\frac{3}{5 m}\right)^{2}}} \rightarrow e \text { and } \\
& D C\left(t_{m}\right)=D\left(e^{\frac{3}{5 m}}\right)=e^{\left(e^{\frac{3}{5 m}}\right)^{2}} \rightarrow e \text { and also } \\
& C D\left(t_{m}\right) \rightarrow e=D(1), D C\left(t_{m}\right) \rightarrow e=C(1) \text { as } m \rightarrow \infty .
\end{aligned}
$$

Therefore $\lim _{n \rightarrow \infty} F_{C D t_{n}, D(1), a}(\beta)=1$ and $\lim _{n \rightarrow \infty} F_{D C t_{n}, C(1), a}(\beta)=1$

$\forall a \in X$ and $\beta>0$.

As a result the pair $(\mathrm{C}, \mathrm{D})$ is conditional semi compatible more over at $\mathrm{t}=0, C(0)=D(0)=1, C D(0)=C(1)=e=D(1)=$ $D C(0)$

implies that the pair $(\mathrm{C}, \mathrm{D})$ is strong semi compatible.

\subsubsection{Example}

Strong semi compatible pair (C, D) of self-mappings do not have to be weakly compatible. Take (X, R) and d denotes the distance on $\mathrm{X}$ and for each $t_{1} \in[0,1]$

$$
\begin{aligned}
& F_{\alpha, \beta, a}\left(t_{1}\right)=\left\{\begin{array}{c}
\frac{t_{1}}{t_{1}+d(\alpha, \beta)}, t_{1}>0 \\
0, t_{1}=1
\end{array}\right. \\
& \forall \alpha, \beta \text { in } X \text { and fixed } a, t_{1}>0 . \\
& \text { define mapping } C, D: X \rightarrow X \text { as } \\
& C(x)=e^{2 x} \quad \forall x \in R \text { and } D(x)=e^{2 x^{2}} \quad \forall x \in R . \\
& \text { choose a sequence }\left(t_{m}\right)=1-\frac{5}{m} \quad \forall m \geq 1 .
\end{aligned}
$$

Then $C\left(t_{m}\right)=C\left(1-\frac{5}{m}\right)=e^{2\left(1-\frac{5}{m}\right)} \quad \rightarrow e^{2}$

and $D\left(t_{m}\right)=D\left(1-\frac{5}{m}\right)=e^{2\left(1-\frac{5}{m}\right)^{2}} \rightarrow e^{2}$, as $m \rightarrow \infty$.

Implies $\left\{\left\langle\left\langle t_{m}\right\rangle: \lim _{m \rightarrow \infty} C t_{m}=\lim _{m \rightarrow \infty} D t_{m}\right\} \neq \emptyset\right.$.

Then there exists another sequence $\left\langle t_{m}\right\rangle=\frac{2}{m} \quad \forall m \geq 1$ Then

$$
\begin{aligned}
& C\left(t_{m}\right)=C\left(\frac{2}{m}\right)=e^{2\left(\frac{2}{m}\right)} \rightarrow 1, \\
& D\left(t_{m}\right)=D\left(\frac{2}{m}\right)=e^{2\left(\frac{2}{m}\right)^{2}} \rightarrow 1 \text { as } m \rightarrow \infty .
\end{aligned}
$$

And also

$$
\begin{aligned}
& C D\left(t_{m}\right)=C\left(e^{\frac{8}{m^{2}}}\right)=e^{2 e^{\frac{8}{m^{2}}}} \rightarrow e^{2} \text { and } \\
& D C\left(t_{m}\right)=D\left(e^{\frac{4}{m}}\right)=e^{2\left(e^{\frac{4}{m}}\right)^{2}} \rightarrow e^{2} . \\
& C D\left(t_{m}\right) \rightarrow e^{2}=D(1), \\
& D C\left(t_{m}\right) \rightarrow e^{2}=C(1) \text { as } m \rightarrow \infty .
\end{aligned}
$$

This gives $\lim _{n \rightarrow \infty} F_{C D t_{n}, D(1), \alpha}(\beta)=1$ and $\lim _{n \rightarrow \infty} F_{D C t_{n}, C(1), a}(\beta)=1$ $\forall a \in X$ and $\beta>0$.

As a result the pair $(\mathrm{C}, \mathrm{D})$ is conditional semi compatible more over at $\mathrm{t}=0, C(0)=D(0)=1, C D(0)=C(1)=e^{2}=D(1)=$ $D C(0)$

implies pair $(C, D)$ is strong semi compatible.

Further at coincidence point $t=1, C(1)=e^{2}=D(1)$ and $C D(1)=C\left(e^{2}\right)=e^{2 e^{2}}, D C(1)=D\left(e^{2}\right)=e^{2 e^{4}}$.

Resulting that the pair $(\mathrm{C}, \mathrm{D})$ is strong semi compatible but not weakly compatible. 


\subsection{Definition ${ }^{(12)}$}

Self-mappings $C, D$ in $(X, F, t)$ are called sub sequentially continuous if there exists a sequence $\left\langle t_{n}\right\rangle \in X$ in order for $C t_{n}, D t_{n} \rightarrow w^{1}$ as $\mathrm{n} \rightarrow \infty$ for certain $w^{1} \in X$ and satisfying

$\lim _{n \rightarrow \infty} F_{C D t_{n}, D w^{1}, a}(\beta)=1$ and $\lim _{n \rightarrow \infty} F_{D C t_{n}, C w^{1}, a}(\beta)=1 \quad \forall a \in X$ and $\beta>0$.

\subsubsection{Example}

Here we give the example in which the $(C, D)$ pair is sub sequentially continuous but not weakly compatible

Let $X=R$ and $\mathrm{d}$ be usual distance on $X$ and for each $t_{1} \in[0,1]$.

Define

$F_{\alpha, \beta, a}\left(t_{1}\right)=\left\{\begin{array}{c}\frac{t_{1}}{t_{1}+d(\alpha, \beta)}, t_{1}>0 \\ 0, t_{1}=1\end{array}\right.$

$\forall \alpha, \beta$ in $\mathrm{X}$ and fixed $a, t_{1}>0$.

Define mappings $C, D: X \rightarrow X$ as

$C(t)=e^{t^{2}} \forall t \in R$ and $D(t)=e^{\frac{t}{2}} \forall t \in R$.

Choose a sequence $\left\langle t_{m}\right\rangle=\frac{2}{m^{2}} \quad \forall m \geq 1$. Then

$C\left(t_{m}\right)=C\left(\frac{2}{m^{2}}\right)=e^{\left(\frac{2}{m^{2}}\right)^{2}} \rightarrow 1 \quad$ and

$D\left(t_{m}\right)=D\left(\frac{2}{m^{2}}\right)=e^{\frac{1}{2}\left(\frac{2}{m^{2}}\right)} \rightarrow 1$ as $m \rightarrow \infty$.

Moreover

$C D\left(t_{m}\right)=C\left(e^{\frac{1}{m^{2}}}\right)=e^{\left(e^{\frac{1}{m}}\right)^{2}} \rightarrow e=C(1) \Rightarrow C D\left(t_{m}\right) \rightarrow C(1)$

$D C\left(t_{m}\right)=D\left(e^{\frac{4}{m^{4}}}\right)=e^{\frac{1}{2} e^{\frac{4}{m^{4}}}} \rightarrow e^{\frac{1}{2}}=D(1) \Rightarrow D C\left(t_{m}\right) \rightarrow D(1)$ as $m \rightarrow \infty$.

This gives $\lim _{n \rightarrow \infty} F_{C D t_{n}}, C(1), a(\beta)=1$ and $\lim _{n \rightarrow \infty} F_{D C t_{n}}, D(1), a(\beta)=1$

$\forall a \in X$ and $\beta>0$

Moreover $C(0)=D(0)=1 \Rightarrow C D(0) \neq D C(0)$ since

$C D(0)=C(1)=e, e^{\frac{1}{2}}=D(1)=D C(0)$

Implies the $(C, D)$ pair is sub sequentially continuous but not weakly compatible.

The following theorem was proved by V. K. Gupta et.al.

Theorem $(\mathrm{A})^{(11)}$ " Let $\mathrm{A}, \mathrm{B}, \mathrm{S}$ and T be self-mappings on a complete probabilistic 2-metric space $(X, F, t)$ satisfying:

$\left(A_{1}\right) A(X) \subseteq T(X), B(X) \subseteq S(X)$

$\left(A_{2}\right)$ one of $A(X), B(X), T(X)$ or $S(X)$ is closed

$\left(A_{3}\right)$ pairs $(A, S)$ and $(B, T)$ are weakly compatible $\left(A_{4}\right) F_{A x . B y, a}(\mathrm{t}) \geq \mathrm{r} F_{S x . T y, a}(\mathrm{t})$ for all x, y and $\mathrm{t}>0$

where $r:[0,1]->[0,1]$ is some continuous function such that $r(t)>t$ for each $0<t<1$

then $\mathrm{A}, \mathrm{B}, \mathrm{S}$ and $\mathrm{T}$ have unique common fixed point in X."

The above Theorem (A) can be further generalized as under.

\section{Results and Discussion}

\subsection{Theorem}

Let $\mathrm{A}, \mathrm{B}, \mathrm{S}$ and $\mathrm{T}$ be self -mappings on a complete probabilistic 2-metric space $(X, F, t)$ satisfying

(3.1.1) $A(X) \subseteq T(X), B(X) \subseteq S(X)$

(3.1.2) pair $(A, S)$ is strongly semi compatible and sub sequentially continuous and the pair $(B, T)$ is strongly semi compatible

(3.1.3) $F_{\mathrm{Ax} . \text { By }, a}(t) \geq \mathrm{r}\left(F_{S x . \mathrm{T} y, a}(t)\right), t>0$ and for all values of $\mathrm{x}, \mathrm{y}$ in $\mathrm{X}$

where $r$ defined on $[0,1]$ to it -self be continuous function with $r(t)>t$ for each $0<\mathrm{t}<1$.

Then $A, B, S$ and $T$ have unique common fixed point in $X$. 
Proof: Take $x_{0} \in X$ and $A x_{0} \in A(X) \subseteq T(X)$ then $\exists x_{1} \in X$ with $A x_{0}=T x_{1}$ having $B x_{1} \in B(X) \subseteq S(X)$ then $\exists x_{2} \in$ $X$ with $B x_{1}=S x_{2}$. Recursively obtain the sequences $\left\langle y_{n}\right\rangle$ and $\left\langle x_{n}\right\rangle$ for $n \geq 1$ such that $\left\langle y_{2 n}\right\rangle=\mathrm{A} x_{2 n}=\mathrm{T} x_{2 n+1}$ and $\left\langle y_{2 n+1}\right\rangle=$ $B x_{2 n+1}=\overline{\mathrm{m}} x_{2 n+2}$ Now our claim is to show that $\left\langle y_{n}\right\rangle$ be a cauchy sequence.

For this take $x=x_{2 n}, y=x_{2 n+1}$ in (3.1.3) we get

$F_{\mathrm{Ax}_{2 n}, B x_{2 n+1, a}}(\mathrm{t}) \geq r F_{S x_{2 n}, T x_{2 n+1}, a}(\mathrm{t})$ this gives

$F_{y_{2 n},}, \quad y_{2 n+1, a}(\mathrm{t}) \geq r F_{y_{2 n-1} \cdot y_{2 n, a} a}(\mathrm{t})>F_{y_{2 n-1} \cdot y_{2 n, a}}(\mathrm{t})$.

Similarly

$F_{y_{2 n+1}, \quad y_{2 n+2, a}}(t)>F_{y_{2 n}, y_{2 n+1, a}}(t)$.

In general $F_{y_{n+1}, y_{n}, a}(t)>F_{y_{n}, y_{n-1, a}}(t)$ for all $n \geq 0$.

Then $\left\langle F_{y_{n+1}} \cdot y_{n}, a(\mathrm{t})>\right.$ for all $n \geq 0$ is an increasing sequence bounded above by 1 .

Therefore it must converges to the limit $L \leq 1$ as $n \rightarrow \infty$

In case of $L<1$ we have $F_{y_{n+1}, y_{n}, a}(t)=L>r(1)>1$ this is absurd. Hence $L=1$.

Hence for all $\mathrm{n}$ and $\mathrm{p}$ we have $F_{y_{n+p}, y_{n}, a}(t)=1$.

As a result, the Cauchy sequence $\left\langle y_{n}\right\rangle$ in $X$ must converge to point $\mathrm{z} \in X$ because $X$ is a complete space.

Consequently each sub sequence also has the same limit $z$.

That is $A x_{2 n}, S x_{2 n} \rightarrow z$ and $B x_{2 n+1}, T x_{2 n+1} \rightarrow z$ as $n \rightarrow \infty$.

By strongly semi compatibility of the pair $(A, S)$ whenever

$A x_{2 n}, S x_{2 n} \rightarrow z$ implies there exists sequence $\left(t_{n}\right) \in X$ such that

$A t_{n}, S t_{n} \rightarrow \bar{u}, \bar{u} \in X$ with

$\lim _{n \rightarrow \infty} F_{\mathrm{ASt}_{n}}, \mathrm{su}, a(t)=1$ and $\lim _{n \rightarrow \infty} F_{\mathrm{SAt}_{n}, \mathrm{~A} \bar{u}, a}(t)=1$ for all $t, a>0 \ldots$

Furthermore, the fact that sub sequentially continuous pair $(A, S)$ implies $\lim _{n \rightarrow \infty} F_{\mathrm{AS} t_{n}, \mathrm{~A} \bar{u}, a}(t)=1$ and $\lim _{n \rightarrow \infty} F_{\mathrm{SA} t_{n}, \mathrm{~S} \bar{u}, a}(t)=1$ for all $t, a>0 \ldots a(3.2)$

Using (3.1) and (3.2) we obtain $F_{\mathrm{A} \bar{u} . s \bar{u} a}(t)=1$ this implies $A \bar{u}=S \bar{u}$

From (3.1.1) $A(X) \subseteq T(X), S \bar{u}=A \bar{u} \in A(X) \subseteq T(X)$ implies

$\exists v_{1} \in X$ such that $S \bar{u}=A \bar{u}=T v_{1}$ for some $v_{1} \in X$.

Claim $B v_{1}=T v_{1}$.

Put $x=\bar{u}, y=v_{1}$ in (3.1.3) we have get

$F_{\mathrm{A} \bar{u}, B v_{1}, a}(\mathrm{t}) \geq \mathrm{r}\left(F_{\mathrm{S} \bar{u} . T v_{1}, a}(\mathrm{t})\right)$ using $S \bar{u}=\mathrm{T} v_{1}$.

$F_{\mathrm{A} \bar{u}, B v_{1} a}(\mathrm{t}) \geq \mathrm{r}\left(F_{\mathrm{S} \bar{u} . \mathrm{S} \bar{u} a}(\mathrm{t})\right)=\mathrm{r}(1)=1$

Hence $A \bar{u}=B v_{1}$ results $A \bar{u}=S \bar{u}=T v_{1}=B v_{1}=P^{1}$ for some $P^{1}$ in $X$.

However, because the pair $(A, S)$ is strongly semi compatible implies $A \bar{u}=S \bar{u} \Rightarrow A S \bar{u}=S A \bar{u} \Rightarrow A P^{1}=S P^{1}$.

Furthermore, the pair $(A, S)$ is strongly semi compatible, resulting in

Errorconverting fromMathMLtoLaTeX

$F_{\mathrm{A} p^{1} . B p^{1} a}(\mathrm{t}) \geq \mathrm{r}\left(F_{S p^{1} . T p^{1} a}(\mathrm{t})\right)$ using $A P^{11}=S P^{1}$ and $B P^{1}=T P^{1}=P^{1}$.

This gives $F_{\mathrm{A}^{1} \cdot p^{1} a}(\mathrm{t}) \geq \mathrm{r}\left(F_{\sigma p^{1} \cdot p^{1} a}(\mathrm{t})\right)$.

If $P^{1} \neq A P^{1}$ implies $F_{\mathrm{A} p^{1} \cdot p^{1} a}(\mathrm{t}) \geq \mathrm{r}\left(F_{\mathrm{A} p^{1} \cdot p^{1} a}(\mathrm{t})\right) .>F_{\mathrm{A} p^{1} \cdot p^{1} a}(\mathrm{t})$.

This gives $F_{\mathrm{A} p^{1} \cdot p^{1} a}(\mathrm{t}) \geq F_{\mathrm{A}^{1} \cdot p^{1} a}(\mathrm{t})$ which is not possible.

Therefore $A P^{1}=S P^{1}=B P^{1}=T P^{1}=P^{1}$.

\section{Uniqueness:}

Let $\mathrm{p}_{1}$ be the another fixed point then 
$A P^{1}=S P^{1}=B P^{1}=T P^{1}=P^{1}$ and $A p_{1}=B p_{1}=S p_{1}=T p_{1}=p_{1}$.

Now assume $P^{1} \neq p_{1}$.

By taking $\mathrm{x}=P^{1}$ and $\mathrm{y}=p_{1}$ in (3.1.3) we've got

$F_{\mathrm{A} p^{1} \cdot B p_{1}} a(\mathrm{t}) \geq \mathrm{r}\left(F_{\mathrm{S}^{1} \cdot \mathrm{T} p_{1} a}(\mathrm{t})\right)$

$F_{p^{1} \cdot p_{1}} a(\mathrm{t}) \geq \mathrm{r}\left(F_{p^{1} \cdot p_{1} a}(\mathrm{t})\right)>F_{p^{1} \cdot p_{1} a}(\mathrm{t})$.

$F_{p^{1} \cdot p_{1} a}(\mathrm{t})>F_{p^{1} \cdot p_{1} a}(\mathrm{t})$ which is not possible hence $P^{1}=p_{1}$.

Now we provide a supporting illustration to justify the theorem.

\subsection{Example}

Consider $\mathrm{X}=[0,1] \mathrm{d}$ is general distance in $\mathrm{X}$ and each $t_{1} \in[0,1]$ take the projection, define

$$
\begin{aligned}
& F_{\alpha, \beta, a}\left(t_{1}\right)=\left\{\begin{array}{c}
\frac{t_{1}}{t_{1}+d(\alpha, \beta)}, t_{1}>0 \\
0, t_{1}=1
\end{array}\right. \\
& A(x)=B(x)=\left\{\begin{array}{c}
\frac{1}{2}, x=0 \\
1-7 x, 0<x \leq \frac{1}{9} \\
x^{2}, \frac{1}{9}<x \leq 1
\end{array}\right. \\
& A(x)=B(x)=\left\{\begin{array}{c}
\frac{1}{3}, x=0 \\
2 x, 0<x \leq \frac{1}{9} \\
x^{4}, \frac{1}{9}<x \leq 1
\end{array}\right.
\end{aligned}
$$

$\operatorname{NowA}(X)=\left(\frac{1}{81}, 1\right]=B(X)$ and $\mathrm{S}(X)=(0,1]=T(X) \operatorname{sothat} A(X) \subseteq \mathrm{T}(X) \operatorname{and} B(X) \subseteq \mathrm{S}(X)$.

Clearly $1 / 9$ and 1 are coincidence points for the mappings $A$, S.

At $x=\frac{1}{9}, A\left(\frac{1}{9}\right)=S\left(\frac{1}{9}\right)=\frac{2}{9}$ and $A S\left(\frac{1}{9}\right)=A\left(\frac{2}{9}\right)=\frac{4}{81}$.

$S A\left(\frac{1}{9}\right)=S\left(\frac{2}{9}\right)=\left(\frac{2}{9}\right)^{4}$ implies $A S\left(\frac{1}{6}\right) \neq S A\left(\frac{1}{6}\right)$.

Consider a sequence $\left\langle x_{n}\right\rangle=\frac{1}{9}-\frac{3}{n}$ for all $n \geq 1$. Then

$$
\begin{aligned}
& A x_{n}=A\left(\frac{1}{9}-\frac{3}{n}\right)=1-7\left(\frac{1}{9}-\frac{3}{n}\right) \rightarrow \frac{2}{9} . \\
& S x_{n}=S\left(\frac{1}{9}-\frac{3}{n}\right)=2\left(\frac{1}{9}-\frac{3}{n}\right) \rightarrow \frac{2}{9} \text { as } n \rightarrow \infty .
\end{aligned}
$$

Implies $\lim _{n \rightarrow \infty} A x_{n}=\lim _{n \rightarrow \infty} S x_{n}$ is nonempty.

There exist another sequence $\left\langle x_{n}\right\rangle=1-\frac{1}{n^{3}}$ for all $n \geq 1$. Then

$A S x_{n}=A S\left(1-\frac{1}{n^{3}}\right)=A\left(1-\frac{1}{n^{3}}\right)^{4}=\left(1-\frac{1}{n^{3}}\right)^{8} \rightarrow 1=S(1)$,

$S A x_{n}=S A\left(1-\frac{1}{n^{3}}\right)=S\left(1-\frac{1}{n^{3}}\right)^{4}=\left(1-\frac{1}{n^{3}}\right)^{8} \rightarrow 1 \rightarrow 1=A(1)$ as $n \rightarrow \infty$.

This implies $\lim _{n \rightarrow \infty} F_{\mathrm{AS} x_{n}}, \mathrm{~s}(1), a(\beta)=1$ and $\lim _{n \rightarrow \infty} F_{\mathrm{SA} x_{n}}, \mathrm{~A}(1), a^{(\beta)}=1$

$\forall a \in X$ and $\beta>0$.

As a result, Pair $(A, S)$ is strong semi-compatible.

Further for the sequence $\left\langle x_{n}\right\rangle=\left(1-\frac{1}{n^{2}}\right)$ for all $\mathrm{n} \geq 1$. Then we have

$$
\begin{aligned}
& A S x_{n}=A S\left(1-\frac{1}{n^{2}}\right)=A\left(1-\frac{1}{n^{3}}\right)^{4}=\left(1-\frac{1}{n^{3}}\right)^{8} \rightarrow 1=A(1) \\
& S A x_{n}=S A\left(1-\frac{1}{n^{2}}\right)=\mathrm{S}\left(1-\frac{1}{n^{3}}\right)^{4}=\left(1-\frac{1}{n^{3}}\right)^{8} \rightarrow 1=S(1) \text { as } n \rightarrow \infty
\end{aligned}
$$

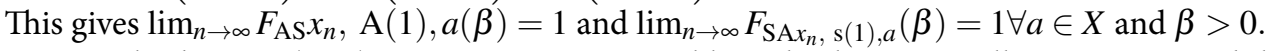

As a result, the pair $(A, S)$ is strong semi compatible and sub sequentially continuous, while the pair $(B, T)$ is strong semi compatible and at $\mathrm{x}=1$, the pair $(B, T)$ is strong semi compatible and

$A(1)=S(1)=B(1)=T(1)=1$

As a result, mappings A, S, B and T met all of the conditions in Theorem (3.1) while also contained the single common fixed point at $\mathrm{x}=1$. 


\subsubsection{Corollary}

In the theorem (3.3) take $T=S$ we get.

Consider $A, B$ and $T$ are self -mappings on a complete probabilistic 2-metric space $(X, F, t)$ having

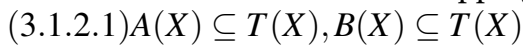

(3.1.2.2) pair $(A, T)$ is strong semi compatible sub sequentially continuous mappings and the pair of mappings $(B, T)$ is faintly compatible

(3.1.2.3) $F_{\mathrm{A} x, B y, a}(\mathrm{t}) \geq \mathrm{r} F_{\mathrm{T} x . \mathrm{T} y, a}(\mathrm{t})$ for all values of $\mathrm{x}, \mathrm{y}$ in $X$ and $t>0$

where some continuous function $\mathrm{r}:[0,1]->[0,1]$ in order for $\mathrm{r}(\mathrm{t})>\mathrm{t}$ for every $t \in(0,1)$ then $\mathrm{A}, \mathrm{B}$ and $\mathrm{T}$ have unique common fixed point in $\mathrm{X}$.

\subsubsection{Corollary}

In Theorem (3.3) take $\mathrm{T}=\mathrm{S}$ and $\mathrm{B}=\mathrm{A}$ we get.

Let $\mathrm{A}, \mathrm{T}$ are self -mappings on a complete probabilistic 2-metric space $(X, F, t)$ satisfying

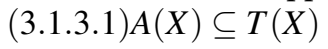

(3.1.3.2) Pair $(A, T)$ is strong semi compatible sub sequentially continuous

(3.1.3.3) $F_{\mathrm{Ax} x \text {. A } y, a}(\mathrm{t}) \geq \mathrm{r}\left(F_{\mathrm{T} x . \mathrm{T} y, a}(\mathrm{t})\right) \forall \mathrm{x}, \mathrm{y}$ and $t>0$

where some continuous function $\mathrm{r}:[0,1]->[0,1]$ in order for $\mathrm{r}(\mathrm{t})>\mathrm{t}$ for every $t \in(0,1)$ then $\mathrm{A}, \mathrm{T}$ have unique common fixed point in X.

\section{Conclusion}

We improved the result in Theorem (A) in this article by applying the concepts of strong semi compatible, sub sequentially continuous mappings and none of the mappings are assumed as closed range spaces. Further we supported our result by discussing a suitable example.

\section{References}

1) Karl M. Statistical metrics. Proceedings of the National Academy of Sciences of the. 1942;28(12):535. Available from: https://doi.org/10.1073/pnas.28.12.535.

2) Dhananjay G, Kumam P, Abbas M. Background and Recent Developments of Metric Fixed Point Theory. 2017.

3) Mishra SN. Common fixed points of compatible mappings in PM-spaces. Math Japon. 1931;36:283-289. Available from: https://ci.nii.ac.jp/naid/ 10010237904/.

4) Martinez-Moreno J, Gopal D, Perov A. Version of Fuzzy Metric Spaces and Common Fixed Points for Compatible Mappings. Mathematics . $2021 ; 9: 1290$. Available from: https://dx.doi.org/10.3390/math9111290.

5) Bijendra S, Shishir J. Semi-compatibility and fixed point theorems in Menger Space. Journal of the Chungcheong Mathematical Society. 2004;17(1):1-7. Available from: http://www.ccms.or.kr/data/pdfpaper/jcms171/1711.pdf.

6) Zhou M, Jain MK, Khan MS. Semi-compatible mappings and common fixed point theorems of an implicit relation via inverse \$C-\$ class functions. AIMS Mathematics . 2021;6(3):2636-2652. Available from: .https://doi.org/10.3934/math.678.2021160.

7) Sunny C, Suneel K, Pant BD. Common fixed point theorems for occasionally weakly compatible mappings in Menger spaces. Journal of Advanced Research in Pure Mathematics. 2021;3(4):17-23. Available from: https://doi.org/10.5373/jarpm.678.122010.

8) Patel DK, Kumam P, Gopal D. Some discussion on the existence of common fixed points for a pair of maps. Fixed Point Theory and Applications . 2013;2013(1):1-17. Available from: https://doi.org/10.1186/1687-1812-2013-187.

9) Mukesh KJ, Saeed KM. Generalization Of Semi Compatibility With Some Fixed Point Theorems Under Strict Contractive Condition. Applied Mathematics E-Notes. 2017;1(17):25-35. Available from: http://www.math.nthu.edu.tw/amen/.

10) Ravindra KB, Naseer S. Faintly compatible mappings and common fixed Points. Fixed point theory and applications. 2013;2013(1):1-19. Available from: https://doi.org/10.1186/1687-1812-2013-156.

11) Arihant J, Gupta VK, Rajesh K. Fixed Point Theorem using Absorbing Mappings in Fuzzy Metric Space. Annals of Pure and Applied Mathematics. 2019;19(2):161-167. Available from: https://doi.org/10.22457/apam.609v19n2a4.

12) Srinivas V, Satyanna K. Some results in Menger space by using sub compatible, faintly compatible mappings. Malaya Journal of Matematik. 2021;9(1):725730. Available from: https://dx.doi.org/10.26637/mjm0901/0127.

13) Satyanna K, Srinvas V. Fixed point theorem using semi compatible and sub sequentially continuous mappings in Menger space. Journal of Mathematical and Computational Science . 2020;10(6):2503-2515. Available from: https://doi.org/10.28919/jmcs/4953.

14) Srinivas V, Thirupathi T. A result on Banach space using E.A like property. Indian Journal of Science and Technology. 2020;13(44):4490-4499. Available from: https://dx.doi.org/10.17485/IJST/v13i44.1909.

15) Sharma R, Ansari HA. Some fixed point theorems in an intuitionistic Menger space via C-class and inverse C-class functions. Computational and Mathematical Methods. 2020;2(4):e1090. Available from: https://doi.org/10.1002/cmm4.1090.

16) Zidan AM. Partial Metric Spaces with some Results in Common Fixed Point Theorems. Journal of Function Spaces. 2021;2021:1-9. Available from: https://doi.org/10.1155/2021/5586936. 